\title{
IL-1 Trap prevents and treats gouty arthritis in a new mouse model
}

Systemic inhibition of interleukin (IL)-1 with rilonacept (IL-1 Trap) could be an effective therapy for the pain and inflammation associated with acute gout. As demonstrated in a novel mouse model, the mouse version of the drug (mIL-1 Trap) both prevented and relieved ankle joint synovitis and hyperalgesia induced by monosodium urate (MSU) crystals.

\section{4 ...the findings of this study further elucidate the potential of IL-1 inhibition in gout 77}

\footnotetext{
"Prior work has established that the production of the proinflammatory cytokine IL- $1 \beta$ is stimulated by MSU crystals, which work through the NLRP3 inflammasome," explains Richard Torres, one of the study's investigators. Experimental models of gouty inflammation have shown that neutrophil influx in response to MSU crystals is reduced in mice deficient in inflammasome components or in the IL-1 receptor (IL1-R1), and is also reduced in response to pharmacological blockade of the IL-1 pathway.

To further explore the consequences of IL-1 blockade, the investigators developed a new acute gout model by use of intraarticular injection of MSU crystals into
}

the ankle joint of mice. "One of the novel elements of our approach was our decision to use markers of hyperalgesia and joint inflammation in mice, as we believed these to be more clinicallyrelevant outcomes since the primary complaint of gout patients is severe pain and joint inflammation."

In addition to the acute gout model, crystal-induced peritonitis and subcutaneous air pouch models as well as genetic depletion of IL-1 were used to study the effects of IL-1 inhibition. "We demonstrated that both $I l 1 r 1^{-/-}$mice and mice pre-treated with mIL-1 Trap showed reduced neutrophil influx in MSU and calcium pyrophosphate dihydrate crystalinduced peritonitis and air pouch models," says Torres. In the acute gout model, knockout mice showed less thermal hyperalgesia and ankle inflammation than wild-type mice, as did mice pretreated with mIL-1 Trap in comparison with vehicle-treated mice.

Both genetic deletion of IL-1R1 and IL-1 inhibition with mIL-1 Trap attenuated MSU-induced up-regulation of proinflammatory cytokines and MSU-induced increases in levels of the acute phase protein serum amyloid A.

Cytokine profiling also confirmed that levels of IL-1 $\beta$ were increased in ankle joints injected with MSU crystals; surprisingly, however, IL-1a levels decreased following MSU crystal injection. This finding lends further support to the idea that IL- $1 \beta$, not IL- $1 \alpha$, drives pain and inflammation in this gout model.

mIL-1 Trap worked as well as highdose colchicine in both preventing and treating neutrophil influx, pain, and ankle inflammation in the acute gout model, although the two treatments do not necessarily have a similar mechanism. "Colchicine is a drug used for both the pain and inflammation in patients with acute gout," says Torres. "It is, however, generally under-utilized by physicians or not adequately adhered to by patients due to severe gastrointestinal toxic effects."

Rilonacept is approved by the FDA for the treatment of cryopyrin-associated periodic syndromes, a group of inflammatory diseases also related to the stimulation of the NLRP3 inflammasome, and the findings of this study further elucidate the potential of IL-1 inhibition in gout. "The data presented in this work suggest that rilonacept may be a promising drug for the prevention and treatment of acute gout attacks," says Torres.

Sarah Price

Original article Torres, R. et al. Hyperalgesia, synovitis, and multiple biomarkers of inflammation are suppressed by IL-1 inhibition in a novel animal model of gouty arthritis. Ann. Rheum. Dis. doi:10.1136/ard.2009.109355 